Joseph's abiding curiosity about the relations between heat and mechanical work.

Clearly, it is hard to know just how farseeing these wider reflexions were. Unlike his better-educated brother, Joseph was a doer, a self-taught inventor, rather than a savant. But it seems likely that from the 1780 s until his death in 1810 , he groped some way towards the notion that heat and work are interchangeable. This, at all events, was the retrospective view of the engineer Marc Seguin, a great-nephew of the Montgolfiers who, as a child, had known and learned from Joseph. Gillispie's careful re-examination of the evidence suggests that Joseph's ideas probably originated in his work on an ingenious heat-pump, a device that raised water by means of the heat produced in the rapid explosion of hydrogen or the burning of faggots. Thereafter, it survived through

\section{Megalithic points}

\section{Clive Ruggles}

\section{Echoes of the Ancient Skies: The \\ Astronomy of Lost Civilizations. \\ By E.C. Krupp.}

Harper and Row: 1983. Pp.386. £16.95.

POPULAR books on archaeoastronomy continue to issue forth from the presses once a year or so, although the emphasis has changed distinctly since the 'Stonehenge Decoded' debate of the 1960's which started it all, and again since the more recent controversies over Alexander Thom's theories on megalithic 'science'. The current message is that astronomy is a sacred rather than a 'scientific' activity; and that in studying archaeoastronomy we are exploring the belief systems, ceremonial activities and cosmologies of diverse societies past (here we now include the great literate civilizations) and present (in this case, for 'archaeoastronomy' read 'ethnoastronomy').

The subject matter of Krupp's book (excluding what amounts to a rather incongruous swift potted history of cosmology in the final chapter in order, apparently, merely to make a concluding point about "why we do it") reflects a change in the definition of archaeoastronomy which is all to the good; archaeoastronomy is beginning to find its feet as a respectable branch of archaeology and ethnography. There is talk of how rituals serve to demarcate and regulate time periods (hardly a new point to anthropologists, admittedly) and hence tend to be related to astronomical observations; and of how the extent of astronomical practice can relate to a society's complexity. It is good to see such points stressed at intervals throughout Echoes of the Ancient Skies, and illustrated with a variety of examples.

Yet I feel, such success has been achieved a series of related inventions of which at least one, the hydraulic ram, was favourably viewed by the leading scientists of the capital. I find the historical significance of this work as intriguing as Gillispie obviously does, though it throws up tantalizing possibilities rather than a justification for a major reinterpretation of the prehistory of thermodynamics.

Despite the loose ends, it is hard to imagine that there is very much more to be said on the Montgolfiers and their world. However, quite apart from its scholarly merits, the book can be recommended as a good read. The work of any historian has its drab moments, but the wit and liveliness of Gillispie's text suggest that in this case such moments were few and far between. $\square$

Robert Fox teaches the history of science at the University of Lancaster. At present, he holds a British Academy Readership in the Humanities.

in the face of, rather than owing to, the thematic way in which the material has been organized. The Maya civilization (to take an extreme example) crops up in no less than six different chapters and might arguably have appeared in one or two more. On the other hand, in the 'Skies we Watch' chapter we are taken in the short space of $\mathbf{4 0}$ pages from ancient Egypt to Shang dynasty China, thence to Babylon, pre-conquest Illinois, prehistoric Scotland and Brittany, the Inca in Peru, the Maya in Mexico, and finally back to ancient China.

All this leaves the reader breathless if not a little confused and at times, as one apparently unrelated description follows another, perhaps even slightly bored; this despite Krupp's enthusiasm and readable. style, and the originality and excitement of much of the subject matter. Attempts to draw threads together are too few and far between, and I wonder if, in the end, archaeoastronomy doesn't come over to the average reader primarily as the mere documentation of astronomical practice.

Krupp has to some extent played down controversy in order to present the evidence for ritual astronomy as a coherent whole. This is defensible for a popular book, but care is needed that a new popular bandwagon (albeit less fantastic than previous ones) does not start rolling.

Already ideas of ritual astronomy are running well ahead of the evidence in some areas, notably the megaliths (where serious debate continues, on the basis of extensive new site surveys and new methodology, about the precision and often the very existence of significant astronomical alignments). I feel that Krupp might have emphasized these areas: by doing so his book would have been truer to the spirit of much current serious archaeoastronomical research, and might have attracted a more discerning readership.

Clive Ruggles is a Research Fellow in the Department of Mathematics at the University of Leicester.

\section{Falling apples and leaning towers}

\author{
John D. Barrow
}

\section{Frame of the Universe:}

A History of Physical Cosmology.

By Frank Durham and

Robert $\mathrm{O}$. Purrington.

Columbia University Press: 1983.

Pp.275. \$32.50, £22.

'HISTORY repeats itself; historians repeat each other'; the natural reaction perhaps of someone seeing another medium-brow guide to the history of science. A sufficient number of such surveys already exist to create a form of '1066 and all that' subculture within the subject. Those events that never happened live most vividly in the mind and are much more memorable than those that really did: Newton's falling apple; Archimedes' bath, Galileo and the tower of Pisa - all are cornerstones of the sub-culture.

The authors of the 'Frame of the Universe' have chosen to follow the history of cosmological ideas from ancient to modern times at a level suitable for nonspecialist college courses and general reading. They claim no great novelty in their treatment and erect their frame around the first ancient and Greek astronomers, the Medievals, Copernicus, Galileo and Newton, before moving on to Einstein, modern big bang cosmology and gravitational collapse. Yet, the clarity of presentation and the engaging style of the authors make this an enjoyable book for any scientist to read. Those wishing to pursue subjects in greater depth are provided with an excellent bibliography and detailed notes.

One of the problems with histories of this sort is that our own categories of thought so influence the presentation. We view the past solely in terms of the route necessary to reach the (right) answers of the present. The failures are ignored as inessential byproducts of a never-faltering march towards the 'truth'. This 'Whig' approach to the history of science is the one that prevails in the minds of most working scientists with a passing interest in the history of their subject and, although the authors are aware of this snare, they do not make any real effort to avoid it. The other weak point in the overall treatment, which will be disappointing to many students reading the book, is that whereas the authors are very lucid in describing the course of events, they are weak on the explanatory side. They rarely ever ask the interesting question 'Why'. Why, for example, did the Jews take no interest in astrology? What role did their religious beliefs play, and so forth.

In the opening chapters there is a particularly clear discussion of various megalithic 'observatories', including Stone- 
henge. Here, and throughout the authors make good use of clear diagrams and some simple geometry to explain details. In the early chapters they do, to my mind, make too much of the idea that most of the content of related archaic myths is induced by astronomical experiences since these were the only universal experiences of early peoples. If we look at the laws of ancient societies we also find an unexpected homogeneity, yet no one would claim that this was astronomically induced.

The ancients lead inevitably to the Greeks and they to the Medievals. Medieval Science was but Greek science written in Latin. Unfortunately, the nonEuropean cultures are all but excluded from the description; nor do the authors ever dwell on the interesting question as to why science developed so effectively in Europe whilst the early Eastern and Oriental successes faded. Some treatment of non-European scientific development would have been valuable, if only because there have recently been a number of ridiculous publications about the relationship of modern physics to Eastern religions.

The Medievals lead inevitably to chapters on Copernicus and Galileo. We see the end of the medieval mindset: the authority of texts gives way to the authority of observations. But, the new dogma, like the old, also has its problems if applied inflexibly, for Copernicus had no concept of the inevitable experimental error associated with any observation or measurement. The authors show how this blindspot influenced Copernicus' deductions and point out that even Newton and his contemporaries failed to appreciate the relation of theoretical predictions to actual measurements. The Copernican development is a good illustration of the 'Whig' approach, as it always appears as the turning point in the history of astronomy. In retrospect, of course, it is; what is now equally clear is that $D e$ Revolutionibus orbium celestium had negligible influence until the seventeenth century. Few copies of it were sold, and even fewer read in the early years after Copernicus' death. Other great events, like the Portuguese voyages of discovery, entirely overshadowed it.

The next stop is Newton and the Principia. After this there is a leap into modern times. General relativity, the big bang and gravitational collapse make up the last quarter of the book. Although the treatment of these subjects is accurate it is extremely lightweight. More mathematical detail was devoted to explaining Ptolemaic epicycles than special relativity. Also, the brief description of elementary particles and the role of symmetry principles was too brief to be of any value.

Another gap in the development was to ignore the way theories were evaluated and the relationship of cosmology to philosophy. In ancient times these two lines of thought regarding the 'frame of the universe' were inseparable but after Descartes no philosopher made a significant contribution to science. The modern era sees a divide between the two disciplines, each of which cultivate different interests. A discussion of how this divide arose and what now distinguishes the two approaches would have been valuable. Also, the authors ignore discussing another modern development: how science thinks about itself. Most scientists take as their article of faith some version of Popper's naive falsification as a guiding principle. However, others following Duhem have stressed that hypotheses can only be tested in bundles, never in isolation. We can save any cherished

\section{Celestial talk}

\section{A. Rupert Hall}

\section{Telescopes, Tides, and Tactics:} A Galilean Dialogue about the Starry Messenger and Systems of the World. By Stillman Drake.

University of Chicago Press: 1983.

Pp.236. \$22.50, £18.

THE translator of all the genuine Galilean dialogues, Stillman Drake, after presenting his version of Galileo's early work in physics in the form of an imaginary dialogue between three of Galileo's friends under the title Cause, Experiment and Science, now employs the same vehicle to describe the first few years of Galileo's activity as an astronomer.

The scene is Venice, the year 1613. Galileo is in Florence. The learned talk is between Sagredo and Salviati - two friends whom Galileo himself used as expositors - and Paolo Sarpi, a religious and a philosopher, another real friend of Galileo's introduced here because Galileo first outlined his theory of the tides to Sarpi (in 1595) and first stated to him the law of fall (in 1604). The talk, without 'Prithees' and 'Forsooths', is in the modern language of a translator, in a style (to my ear) rather more formal and stilted than that actually employed by Galileo himself. Perhaps as these are imitations of imaginary conversations, that is right. Whether the dialogue form is preferable to straightforward historical exposition is a matter of taste; the facts and arguments are clear enough here, and Drake provides references for those sentences that have been lifted more or less literally from Galileo's own writings.

A substantial part of the book is the 'reading' of Galileo's Starry Messenger (Sidereus Nuncius, 1610), of which Drake published a partial English version many years ago. The printing of a complete translation now, with all the details of Galileo's observations of Jupiter's satellites, enables Drake to give an analysis - part historical, part reconstruction - of Galileo's longest hypothesis from falsification indefinitely by invariably picking as false another member of the 'bundle' involved in the experiment. In fact, the way in which the false assumption is picked from the 'bundle' is largely what separates 'crank' science from real science.

This was a book I enjoyed reading and one which undergraduates in historical subjects should find useful as an introductory guide. British science students suffering from educational overspecialization might also enjoy seeing when and how authority changed.

John D. Barrow is a Lecturer in Astronomy at the University of Sussex.

continued, most exact and most frustrating investigation in observational astronomy, which never yielded that practical solution to the problem of longitude at sea, ardently hoped for by the discoverer. This analysis, in turn, adds further substance to Drake's view of Galileo as, above all, an empirical investigator of nature.

There are many other issues raised in the dialogue, not all of them firmly resolved (it is a real advantage of the conversational vehicle that it allows a degree of uncertainty common in life but rare in historians). It starts from the question: why

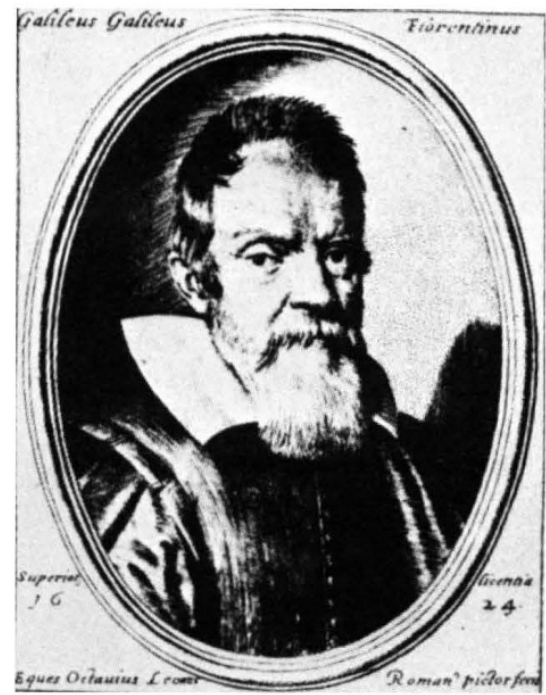

Galileo Galilei

has Galileo not published the System of the World announced in print in 1610 and in private even earlier? This, together with the related theme of the mechanical explanation of the tides, permits Drake to put forward new ideas about Galileo's development as an exponent of the new astronomy - in which context his relationship with Kepler, another theme, is also relevant. In particular Drake introduces the plausible hypothesis that Galileo whom history knows only as a student of motion for his first thirty years and more was led to take Copernicanism seriously by hitting upon his 'inertial' explanation of the tides (1595). This explanation was, 